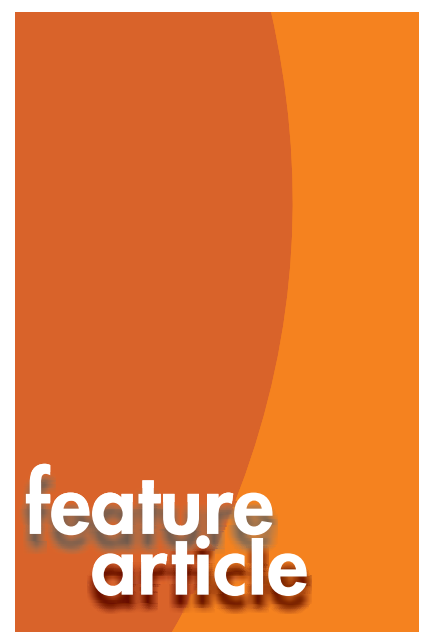

\title{
Shear thickening in colloidal dispersions
}

\author{
Norman J. Wagner and John F. Brady
}

Shampoos, paints, cements, and soft body armor that stiffens under impact are just a few of the materials whose rheology is due to the change in viscosity that occurs when colloidal fluids experience shear stress.

\begin{abstract}
Norm Wagner is the Alvin B. and Julia O. Stiles Professor and chairperson of the department of chemical engineering at the University of Delaware in Newark. John Brady is the Chevron Professor of Chemical Engineering at the California Institute of Technology in Pasadena.
\end{abstract}

The popular interest in cornstarch and water mixtures known as "oobleck" after the complex fluid in one of Dr. Seuss's classic children's books arises from their transition from fluid-like to solid-like behavior when stressed. The viscous liquid that emerges from a roughly 2-to-1 (by volume) combination of starch to water can be poured into one's hand. When squeezed, the liquid morphs into a doughy paste that can be formed into shapes, only to "melt" into a puddle when the applied stress is relieved. Internet videos show people running across a large pool of the stuff, only to sink once they stop in place, and "monsters" that grow out of the mixture when it's acoustically vibrated (for an example, see the online version of this article).

Shear-thickening fluids certainly entertain and spark our curiosity, but their effect can also vex industrial processes by fouling pipes and spraying equipment, for instance. And yet, when engineered into composite materials, STFs can be controlled and harnessed for such exotic applications as shockabsorptive skis and the soft body armor discussed in box 1 .

Engineers and colloid scientists have wrestled with the scientific and practical problems of shear-thickening colloidal dispersions - typically composed of condensed poly- mers, metals, or oxides suspended in a liquid - for more than a century. More recently, the physics community has explored the highly nonlinear materials in the context of jamming $^{1}$ (see the article by Anita Mehta, Gary Barker, and JeanMarc Luck in PHYSICS TODAY, May 2009, page 40) and the more general study of colloids as model systems for understanding soft condensed matter.

Hard-sphere colloids are the "hydrogen atom" of colloidal dispersions. Because of their greater size and interaction times compared with atomic and molecular systems, colloidal dispersions are often well suited for optical microscopy and scattering experiments using light, $\mathrm{x}$ rays, and neutrons. That makes the dispersions, beyond their own intrinsic technological importance, ideal models for exploring equilibrium and near-equilibrium phenomena of interest in atomic and molecular physics - for example, phase behavior and "dynamical arrest," in which particles stop moving collectively at the glass transition. The relevance of colloids to atomic and molecular systems breaks down, though, for highly nonequilibrium phenomena. Indeed, shear thickening in strongly flowing colloidal dispersions may be among the most spectacular, and elucidating, examples of the differences between the systems.

\section{Box 1. Soft armor and other applications}

The unique material properties of increased energy dissipation combined with increased elastic modulus make shearthickening fluids (STFs) ideal for damping and shock-absorption applications. For example, so-called EFiRST fluids can be switched between shear-thickened and flowing states using an applied electric field, which controls the damping. Researchers have also explored the STF response in sporting equipment ${ }^{14}$ and automotive applications, ${ }^{15}$ such as skis and tennis rackets that efficiently dissipate vibrations without losing stiffness or STFs embedded in a passenger compartment liner designed to protect passengers in a car accident.

One commercial application of STF composites is expected to be protective clothing. ${ }^{16}$ The fabric imaged in these scanning electron micrographs has STFs intercalated into its woven yarns. Initial applications are anticipated in flexible vests for correctional officers. Longer-term research is being performed in one of our laboratories (Wagner's), in conjunction with the US Army Research Laboratory, to use STF fabrics for ballistic, puncture, and blast protection for
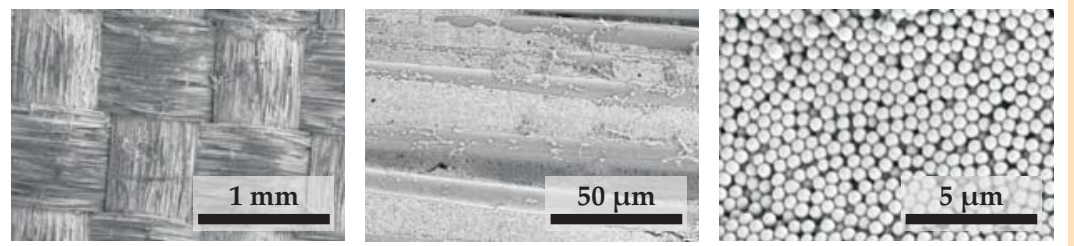


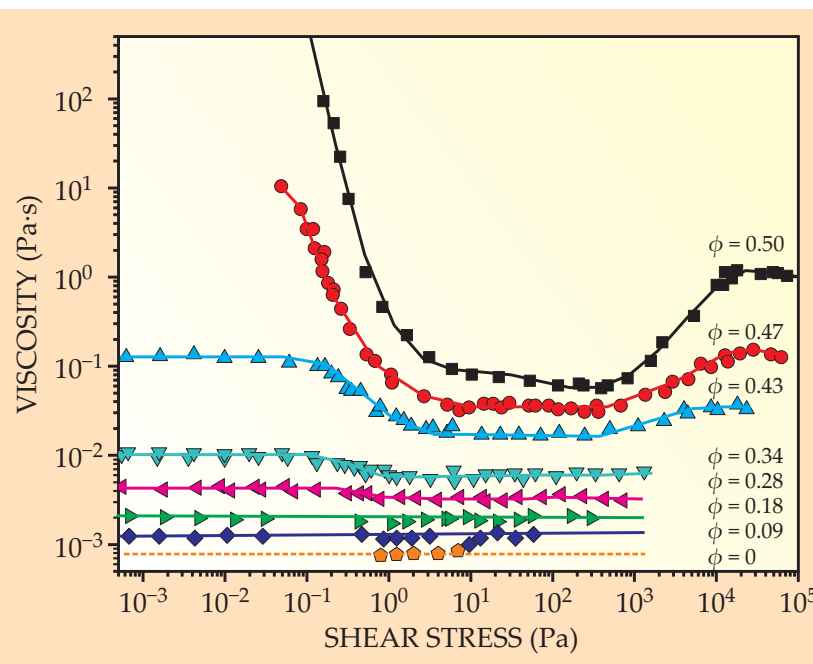

Figure 1. The viscosity of colloidal latex dispersions, as a function of applied shear stress. The volume fraction $\phi$ of latex particles in each dispersion distinguishes the curves. A critical yield stress must be applied to induce flow in a dispersion with high particle concentration. Beyond that critical stress, the fluid's viscosity decreases (shear thinning). At yet higher stress, its viscosity increases (shear thickening), at least for latex dispersions above some concentration threshold. (Adapted from ref. 12.)

Figure 1 illustrates the effect. The addition of colloidal particles to a liquid such as water results in an increase in the liquid's viscosity and, with further addition, the onset of nonNewtonian behavior - the dependence of its viscosity on an applied shear stress or shear rate. At high particle concentrations, the fluid behaves as if it has an apparent yield stress. That is, it must be squeezed, like ketchup, before it can actually flow. At such concentrations, the colloidal dispersions fit into the general paradigm for jamming in soft matter: ${ }^{2}$ At high particle densities and low stresses (and low temperatures, usually), the system dynamically arrests, just as atomic, molecular, polymeric, and granular systems do. But once the yield stress is exceeded, the fluid's viscosity drops, a response known as shear thinning. That rheology is engineered into a range of consumer products, from shampoos and paints to liquid detergents, to make them gel-like at rest but still able to flow easily under a weak stress. Again, the colloid model fits the general paradigm for how matter behaves: It flows when sheared strongly enough.

At higher stresses, shear thickening occurs: Viscosity rises abruptly, sometimes discontinuously, once a critical shear stress is reached. The rise is counterintuitive and inconsistent with our usual experience. Experiments and simulations on atomic and small-molecule liquids predict only shear thinning, at least until the eventual onset of turbulence at flow rates that vastly exceed those of interest here.

The ubiquity of the phenomenon in the flow of suspended solids is a serious limitation for materials processing, especially when it involves high shear-rate operations. In a 1989 review, Howard Barnes writes,

Concentrated suspensions of nonaggregating solid particles, if measured in the appropriate shear rate range, will always show (reversible) shear thickening. The actual nature of the shear thickening will depend on the parameters of the suspended phase: phase volume, particle size (distribution), particle shape, as well as those of the suspending phase (viscosity and the details of the deformation, i.e., shear or extensional flow, steady or transient, time and rate of deformation). ${ }^{3}$

Inks, polymeric binders for paints, pastes, alumina casting slurries, blood, and clays are all known to shear thicken. But the earliest searches for the root cause came from industrial laboratories that coated paper at high speeds (shear rates typically up to $10^{6} \mathrm{~Hz}$ ), a process in which the coating's increasing viscosity would either tear the paper or ruin the equipment. Industrial labs remain intensely interested in the science. Hundreds of millions of metric tons of cement are used globally each year, for example, and production engineers are careful to formulate modern high-strength cements and concretes that don't suffer from the effect-at least in a range of shear rates important for processing and construction. ${ }^{4}$

In pioneering work in the 1970s, Monsanto's Richard Hoffman developed novel light-scattering experiments to probe the underlying microstructural transitions that accompanied shear thickening in concentrated latex dispersions. ${ }^{5}$ The transition was observed to correlate with a loss of Bragg peaks in the scattering measurement. On that basis, Hoffman developed a micromechanical model of shear thickening as a flow-induced order-disorder transition.

In the 1980s and early 1990s BASF's Martin Laun and others interested in products such as paper coatings and emulsion-polymerized materials used then emerging smallangle neutron-scattering techniques to demonstrate that an order-disorder transition was neither necessary nor alone sufficient to induce significant shear thickening. ${ }^{6}$ Because shear thickening is a highly nonequilibrium, dissipative state, though, a full understanding had to await the development of new theoretical and experimental tools.

\section{Hydrodynamics}

The dynamics of colloidal dispersions is inherently a manybody, multiphase fluid-mechanics problem. But first consider the case of a single particle. Fluid drag on the particle leads to the Stokes-Einstein-Sutherland fluctuation-dissipation relationship:

$$
D_{0}=\frac{k T}{6 \pi \mu a} .
$$

The diffusivity $D_{0}$ scales with the thermal energy $k T$ divided by the suspending medium's viscosity $\mu$ and the particle's hydrodynamic radius $a$. That diffusivity sets the characteristic time scale for the particles' Brownian motion; it takes the particle $a^{2} / D_{0}$ seconds to diffuse a distance equal to its radius. The time scale defines high and low shear rates $\dot{\gamma}$.

A dimensionless number known as the Péclet number, $\mathrm{Pe}$, relates the shear rate of a flow to the particle's diffusion rate; alternatively, the Péclet number can be defined in terms of the applied shear stress $\tau$ :

$$
\mathrm{Pe}=\frac{\dot{\gamma} a^{2}}{D_{0}}=\frac{\tau a^{3}}{k T} .
$$

The number is useful because dispersion rheology is often measured by applied shear rates or shear stresses. Low Pe is close enough to equilibrium that Brownian motion can largely restore the equilibrium microstructure on the time scale of slow shear flow. At sufficiently high shear rates or stresses, though, deformation of the colloidal microstructure by the flow occurs faster than Brownian motion can restore it. Shear thinning is already evident around $\mathrm{Pe} \approx 1$. And higher shear rates or stresses (higher Pe) trigger the onset of shear thickening. 
The presence of two or more particles in the suspension fundamentally alters the Brownian motion due to the inherent coupling, or hydrodynamic interaction, between the motion of the particles and the displacement of the suspending fluid. In a series of seminal articles in the 1970s, Cambridge University's George Batchelor laid a firm foundation for understanding the colloidal dynamics. ${ }^{7}$ In essence, because any particle motion must displace incompressible fluid, a longranged-and inherently many-body-force is transmitted from one particle through the intervening fluid to neighboring particles; the result is that all particles collectively disturb the local flow field through hydrodynamic interactions. Such interactions are absent in atomic and molecular fluids, where the intervening medium is vacuum.

Batchelor's calculation of the trajectories of nonBrownian particles under shear flow identified the critical importance of what's known as lubrication hydrodynamics, which describes the behavior of particles interacting via the suspending medium at very close range. Those hydrodynamics were already well known in the fluid mechanics of journal bearings, which Osborne Reynolds investigated in the late 1800 s and which remain of great importance to the workings of modern machines. As box 2 explains, the force required to push two particles together in a fluid diverges inversely with their separation distance. Of particular significance is that at close range, the trajectories that describe their relative motion become correlated. That is, the particles effectively orbit each other-indefinitely if they are undisturbed.

Batchelor's work also led to a formal understanding of how hydrodynamic coupling alters the fluctuationdissipation relationship, which, in turn, enabled him to calculate the diffusion coefficient and viscosity of dilute dispersions of Brownian colloids at equilibrium. ${ }^{7}$ Although it was not fully appreciated at the time, the effect of hydrodynamic interactions on particle trajectories is the basis for understanding the shear-thickening effect.

\section{Beyond two particles}

Hydrodynamic interactions in real colloidal suspensions require numerical methods to solve. The method of Stokesian dynamics outlined in box 3 calculates the properties of ensembles of colloidal and noncolloidal spheres under flow. A great advantage of the simulations is their ability to resolve which forces contribute to the viscosity. Moreover, they demonstrate that the ubiquitous shear thinning in hard-sphere colloidal dispersions is a direct consequence of particle rearrangement due to the applied shear.

The equilibrium microstructure is set by the balance of stochastic and interparticle forces at play-including electrostatic and van der Waals forces - but is not affected by hydrodynamic interactions. The low-shear $(\mathrm{Pe} \ll 1)$ viscosity has two components, one due to direct interparticle forces, which dominate, and one due to hydrodynamic interactions. ${ }^{7}$ Under weak but increasing shear flow (Pe $\sim 1)$, the fluid structure becomes anisotropic as particles rearrange to reduce their interactions so as to flow with less resistance. Figure 2 illustrates the evolution schematically. Near equilibrium, the resistance to flow is naturally high because shearing the random distribution of particles causes them to frequently collide, like cars would if careening haphazardly along a road. With increasing shear rates, though, particles behave as if merging into highway traffic: The flow becomes streamlined and the increasingly efficient transport of colloidal particles reduces the system's viscosity.

Simulations that ignore hydrodynamic coupling between particles show that the ordered, low-viscosity state persists even as the Péclet number approaches infinity. Think of particles sliding by in layers orthogonal to the shear-

\section{Box 2. Lubrication hydrodynamics and hydroclusters}

When two colloidal particles approach each other, rising hydrodynamic pressure between them squeezes fluid from the gap. At close range, the hydrodynamic force increases inversely with the distance between the particles' surfaces and diverges to a singularity. The graph at right plots the normalized force required to drive two particles together (along a line through their centers) at constant velocity. The Navier-Stokes equations that govern the flow behavior between particles are time reversible, so the force is the same one required to separate two particles.

In simple shear flow, particle trajectories are strongly coupled by the hydrodynamic interactions if the particles are close together. The inset of the plot shows a test particle's trajectories, sketched as

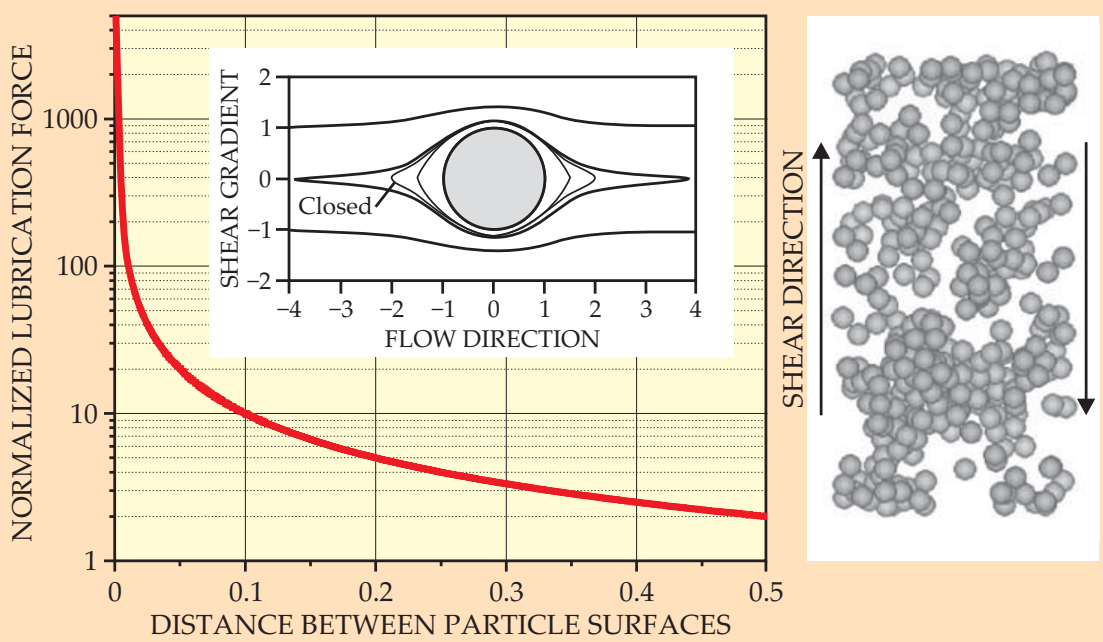
paths as it moves in a shear flow relative to a reference particle (gray sphere). The trajectories are reversible and can be divided into two classes: those that come and go to infinity and those that lead to correlated orbits-so-called closed trajectoriesaround the reference particle.

Simulations and theory of concentrated dispersions that account for those short-range hydrodynamics show that at high shear rates, particles that are driven into close proximity remain strongly correlated and are reminiscent of the closed trajectories observed in dilute suspensions. The flow-induced density fluctuations are known as hydroclusters. Because the particle concentration is higher in the clusters, the fluid is under greater stress, which leads to an increase in energy dissipation and thus a higher viscosity. The illustration at right during a stage of the Stokesian dynamics simulation shows colloidal particles in hydroclusters. ${ }^{8}$ 


\section{Box 3. Stokesian dynamics}

The flow of particles suspended in an incompressible Newtonian fluid is a challenging fluid-mechanics problem that can be handled analytically for a single sphere and semianalytically for two spheres. For three or more particles, though, it requires a numerical solution to the Stokes equation-the Navier-Stokes equation without inertia. Solution strategies range from brute-force finite-element calculations, to more elegant boundary integral methods, to coarse-grained methods, such as smoothedparticle hydrodynamics or lattice Boltzmann techniques, for representing the fluid. The method of Stokesian dynamics ${ }^{17}$ starts with the Langevin equation for $\mathrm{N}$-particle dynamics,

$$
\mathbf{M} \cdot \frac{d \mathbf{U}}{d t}=\mathbf{F}_{\mathrm{P}}+\mathbf{F}_{\mathrm{H}}+\mathbf{F}_{\mathrm{B}}
$$

in which the tensor $\mathbf{M}$ is a generalized mass, a $6 N \times 6 N$ mass and moment-of-inertia matrix; $\mathbf{U}$ is the $6 \mathrm{~N}$-dimensional particle translational and rotational velocity vector; and the $6 \mathrm{~N}$-dimensional force and torque vectors represent the interparticle and external forces $\mathbf{F}_{\mathrm{p}}$ (such as gravity), hydrodynamic forces $\mathbf{F}_{\mathrm{H}}$ acting on the particles due to their motion relative to the fluid, and stochastic forces $\mathbf{F}_{\mathrm{B}}$ that give rise to Brownian motion. The stochastic forces are related to the hydrodynamic interactions through the fluctuation-dissipation theorem.

In Stokes flow the hydrodynamic forces and torques are linearly related to the particle translational and rotational velocities as $\mathbf{F}_{\mathrm{H}}=-\mathbf{R} \cdot \mathbf{U}$, where $\mathbf{R}$ is the configuration-dependent hydrodynamic resistance matrix. In the Stokesian dynamics method, the necessary matrices are computed by taking advantage of the linearity of the Stokes equations and their integral solutions. Long-range many-body hydrodynamic effects are accurately computed by a force-multipole expansion and combined with the exact, analytic lubrication hydrodynamics to calculate the forces.

Armed with that method, one can predict the colloidal microstructure associated with a particular shear viscosity. Take, for instance, a concentrated colloidal dispersion whose particles occupy nearly half the volume. If the positions of those particles are represented as dots, the figure illustrates how the hydrodynamic forces affect their probable locations around some arbitrary test particle (black). The three panels differ only in the shear rate, represented by the Péclet number Pe, the ratio of the shear and diffusion rates.

At low Péclet number (0.1), the distribution of neighboring particles is isotropic, which is typical of a concentrated liquid. Red indicates the most probable particle positions as nearest neighbors and green the least probable. At $\mathrm{Pe}=1$, significant shear distortion appears in neighbor distributions, such that particles are convected together along the compression axes $\left(135^{\circ}\right.$ and $\left.-45^{\circ}\right)$ relative to the shear flow. At high Péclet numbers, the shear-thickening regime, particles aggregate into closely connected clusters, which is manifest as yet greater anisotropy in the microstructure. Particles are more closely packed and thus occupy a narrower region (red) around the test particle than at lower Péclet numbers, indicative of being trapped by the lubrication forces. ${ }^{18}$ gradient direction. Stokesian dynamics simulations, however, demonstrate that hydrodynamic forces become larger at high shear rates $(\mathrm{Pe} \gg 1)$ than do interparticle forces that drive Brownian motion. So when the particles are driven close together by applied shear stresses, lubrication hydrodynamics strongly couple the particles' relative motion. The result is a colloidal dispersion that has a microstructure significantly

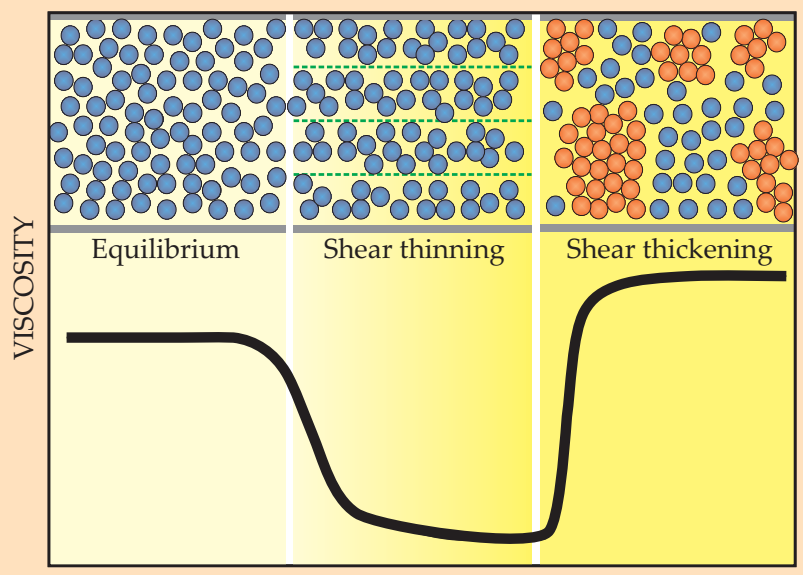

SHEAR STRESS OR SHEAR RATE different from the one near equilibrium, and hence, the energy dissipation increases. In hindsight, that should not be surprising given Batchelor's calculation of closed trajectories.

In both semidilute and concentrated dispersions, the strong hydrodynamic coupling between particles leads to the formation of hydroclusters - transient concentration fluctuations that are driven and sustained by the applied shear field. Here again, the analogy to traffic collisions disrupting organized, low-dissipation flow may be helpful. Unlike the seemingly random microstructure observed close to equilibrium, however, this microstructure is highly organized and

Figure 2. The change in microstructure of a colloidal dispersion explains the transitions to shear thinning and shear thickening. In equilibrium, random collisions among particles make them naturally resistant to flow. But as the shear stress (or, equivalently, the shear rate) increases, particles become organized in the flow, which lowers their viscosity. At yet higher shear rates, hydrodynamic interactions between particles dominate over stochastic ones, a change that spawns hydroclusters (red) - transient fluctuations in particle concentration. The difficulty of particles flowing around each other in a strong flow leads to a higher rate of energy dissipation and an abrupt increase in viscosity. 


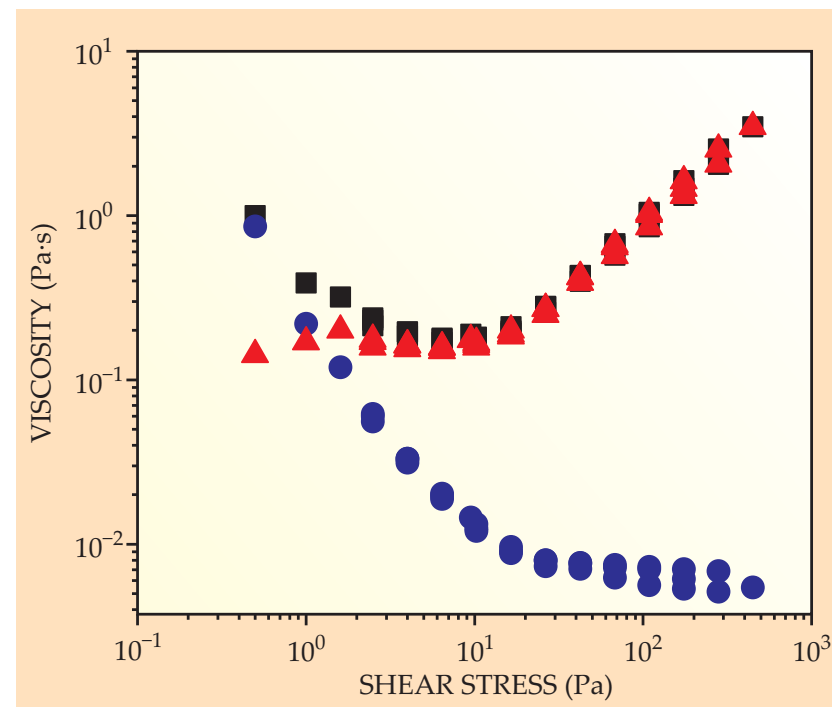

Figure 3. The measured viscosity of a concentrated colloidal suspension (squares) can be resolved into two components-a thermodynamic component (circles) associated with the stochastic motion of particles and a hydrodynamic component (triangles) associated with forces acting between particles due to motion through the suspending fluid. Light-scattering experiments combined with numerical simulations determine which forces dominate in different stress regimes. (Adapted from ref. 13.)

anisotropic. The transient hydroclusters are the defining feature of the shear-thickening state.

Referring back to figure 1, one can see that a colloidal volume fraction $\phi=0.50$ produces a latex dispersion whose viscosity is $1 \mathrm{~Pa} \cdot \mathrm{s}$ at a low shear stress and again at one more than four orders of magnitude higher. The same viscosity emerges for very different reasons, though. Changes in the particles' size, shape, surface chemistry, and ionic strength and in properties of the suspending medium all affect the interparticle forces, which dominate the viscosity at low shear stress. Hydrodynamic forces, in contrast, dominate at high shear stress. Understanding the difference is critical to formulating a dispersion that behaves as needed for specific processes or applications.

As shown in figure 3, rheo-optical measurements on model dispersions experimentally confirm the predictions of

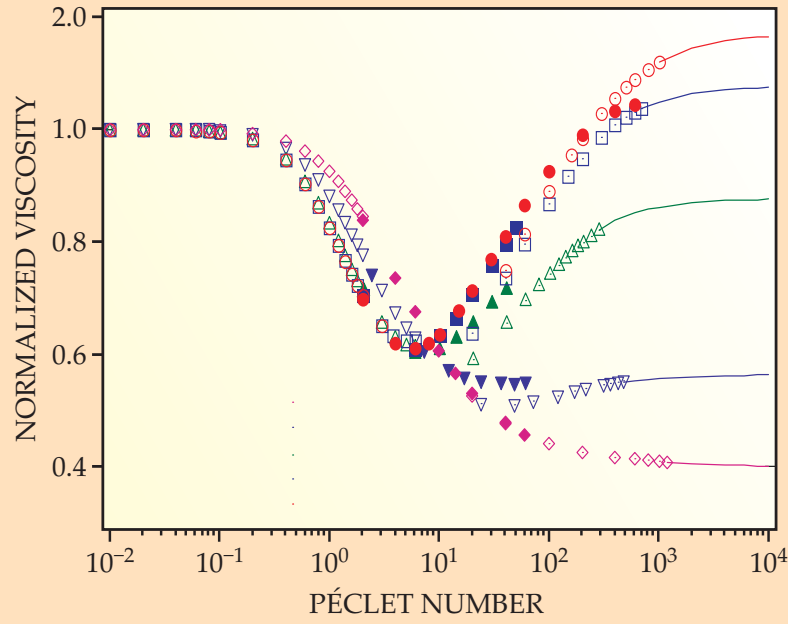

simulations that the shear-thickened state is driven by dissipative hydrodynamic interactions. The flow generates strong anisotropy in the nearest-neighbor distributions (see box 3). The anisotropies give rise to clusters of particles and concomitant large stress fluctuations ${ }^{8}$ that, in turn, lead to high dissipation rates and thus a high shear viscosity. The formation of hydroclusters is generally reversible, though, so reducing the shear rate returns the suspension to a stable, flowing suspension with lower viscosity. Moreover, even very dilute dispersions will shear thicken, although the effect is hard to observe. ${ }^{9}$

Controlling shear thickening requires different strategies from those typically employed to control the low-shear viscosity. The addition, for example, of a polymer "brush" grafted or adsorbed onto the particles' surface can prevent particles from getting close together. With the right selection of graft density, molecular weight, and solvent, the onset of shear thickening moves out of the desired processing regime. ${ }^{10}$ The strategy is often used to reduce the viscosity at high processing rates but could increase the suspension's low-shear viscosity.

Indeed, because the separation between hydroclustered particles is predicted to be on the order of nanometers for typical colloidal dispersions, shear-thickening behavior directly reflects the particles' surface structure and any short-range interparticle forces at play. Fluid slip, adsorbed ions, surfactants, polymers, and surface roughness all significantly influence the onset of shear thickening. Simple models based on the hydrocluster mechanism have proven valuable in predicting the onset of shear thickening and its dependence on those stabilizing forces. ${ }^{11}$

Figure 4 shows a toy-model calculation in which shear thickening is suppressed by imposing a purely repulsive force field-akin to the effect of a polymer brush-around each particle that prevents the particles from getting too close to each other. ${ }^{9}$ When the range of the repulsive force approaches $10 \%$ of the particle radius, the shear thickening is effectively eliminated and the suspension flows with low viscosity. Manipulating those nanoscale forces, the particles' composition and shape, and properties of the suspending fluid so as to control the sheer thickening, however, remains a challenge for the suspension formulator.

\section{Beyond hard spheres}

Although the basic micromechanics of shear behavior in colloidal suspensions are understood, many aspects of the fascinating and complex fluids remain active research problems. At very high particle densities, dispersions can undergo discontinuous shear thickening whereby the suspension will not shear at any higher rate. Rather, increasing the power to a rheometer, for example, leads to such dramatic increases in viscosity and

Figure 4. Shear thickening can be suppressed by reducing the interactions between particles, as shown here based on numerical calculations. The extent of the reduction affects whether an increasing Péclet number (a measure of shear rate) leads to a shear-thickening state or a shear-thinning one. The effect is evident experimentally when a polymer layer, or brush, is grated onto the particles: The dispersion becomes progressively less viscous as the brush thickness on each particle increases. (Adapted from ref. 9.) 


\section{Nanoquest}

\section{Ion Beam Sputter \& Etch System}

- Etch noble metals (Au, Pt, Ru, Pd, Rh)

with simple inert gas

- Etch magnetic materials

- Etch and passivate in same run

- Reactive sputter and etch

- 50 to $200 \mathrm{~mm}$ diameter substrates

- SIMS End-Point detection

- Load-locked operation

- Ion Beam Assisted Deposition (IBAD)

- Water-cooled substrate stage with adjustable spin and rotation and integrated beam probe

INTLWAC A World Leader in lon Beam Technology

Tel+19058730166 • dino@intlvac.com • www.intlvac.com

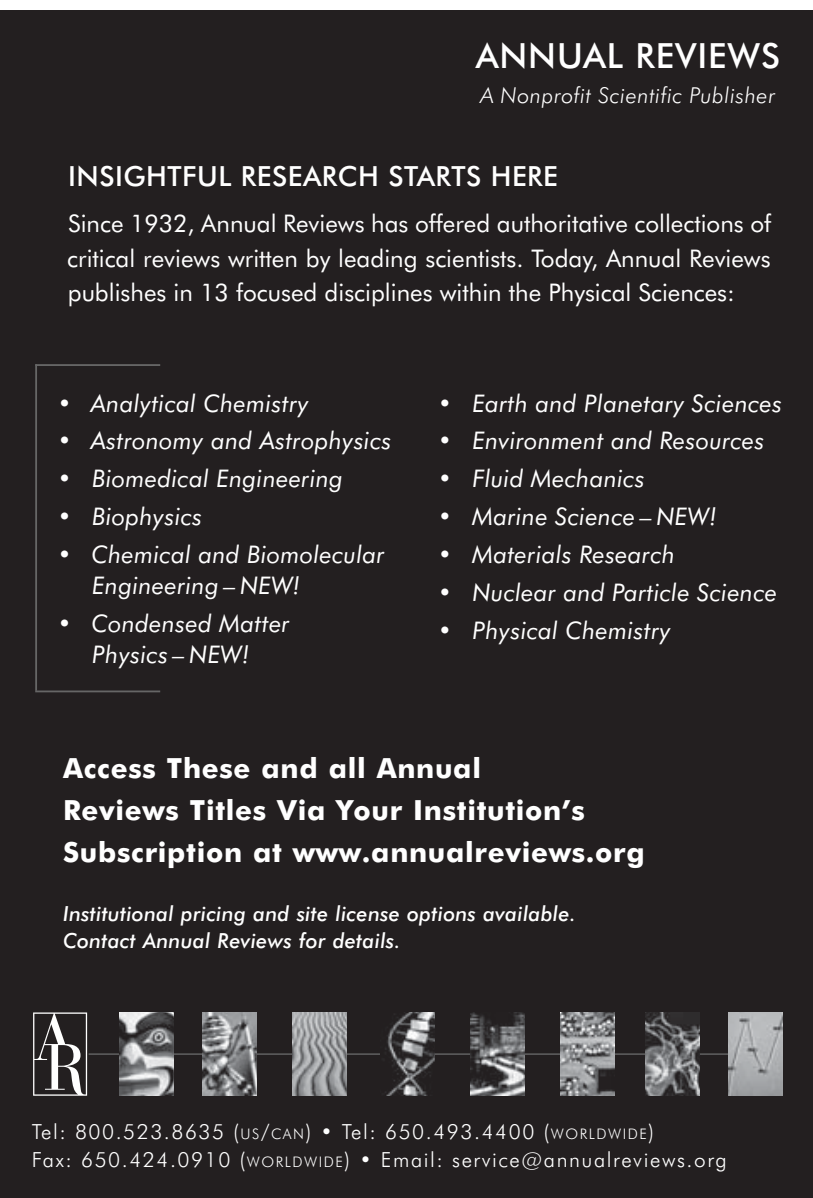

large fluctuations in stress that the suspension either refuses to flow any faster or solidifies. Samples that exhibit strong shear thickening are particularly interesting as candidates for soft body armor (see box 1), and that application has prompted investigations of transient shear thickening at microsecond time scales and at stresses that approach the ideal strength of the particles.

Another active research topic concerns jamming transitions under flow. As figure 1 suggests, concentrated suspensions could be jammed at low and high shear stresses but flow in between. Evidence also exists, as the figure more subtly suggests, that dispersions may exhibit a second regime of shear thinning at the highest stresses rather than continuing to resist the increasing shear rate. The effect can be understood as a manifestation of the finite elasticity of the particles - relatively soft plastic in this case. At very high stresses, particles stop behaving like billiard balls and elastically deform, which alters their rheology. The same forces that drive the hydrocluster formation, which is reversible as the flow is reduced, can also lead to irreversible aggregation. That is, particles forced into contact remain in contact even as the flow weakens. Such shear-sensitive dispersions irreversibly thicken and are often undesirable in practice.

Conversely, in dispersions composed of particle aggregates or fillers such as fumed silica or carbon black, the extreme forces can lead to particle breakage and thixotropy (time-dependent viscosity). Indeed, propagating those forces into the colloids may be key to splitting the colloids into nanoparticles. It's thought, for instance, that the extreme mechanical stress required to grind up and pulverize particles is more effectively transferred to the particles when they are in a shear-thickened state in the slurry of a mill.

Interesting questions arise in the role of shear thickening in chemical mechanical planarization, a critical step in semiconductor processing. Concentrated dispersions are useful for other polishing operations as well, and the control of their shear thickening can be critical to performance.

Although it's impossible to completely survey the science surrounding shear thickening in colloidal dispersions and its applications, we hope the highly counterintuitive rheology has piqued your interest. A wealth of fascinating challenges and applications awaits.

\section{References}

1. C. B. Holmes et al., J. Rheol. 49, 237 (2005).

2. A. J. Liu, S. R. Nagel, Nature 396, 21 (1998).

3. H. A. Barnes, J. Rheol. 33, 329 (1989).

4. F. Toussaint, C. Roy, P.-H. Jézéquel, Rheol. Acta, doi:10.1007/ s00397-009-0362-z (2009).

5. R. L. Hoffman, J. Rheol. 42, 111 (1998).

6. H. M. Laun et al., J. Rheol. 36, 743 (1992).

7. W. B. Russel, D. A. Saville, W. R. Schowalter, Colloidal Dispersions, Cambridge U. Press, New York (1989).

8. J. R. Melrose, R. C. Ball, J. Rheol. 48, 961 (2004).

9. J. Bergenholtz, J. F. Brady, M. Vicic, J. Fluid Mech. 456, 239 (2002).

10. L.-N. Krishnamurthy, N. J. Wagner, J. Mewis, J. Rheol. 49, 1347 (2005).

11. B. J. Maranzano, N. J. Wagner, J. Chem. Phys. 114, 10514 (2001).

12. H. M. Laun, Angew. Makromol. Chem. 123, 335 (1984).

13. J. Bender, N. J. Wagner, J. Rheol. 40, 899 (1996).

14. C. Fischer et al., Smart Mater. Struct. 15, 1467 (2006).

15. H. M. Laun, R. Bung, F. Schmidt, J. Rheol. 35, 999 (1991).

16. Y. S. Lee, E. D. Wetzel, N. J. Wagner, J. Mater. Sci. 38, 2825 (2003).

17. For a review, see J. F. Brady, G. Bossis, Annu. Rev. Fluid Mech. 20, 111 (1988).

18. D. R. Foss, J. F. Brady, J. Fluid Mech. 407, 167 (2000). 